\title{
Comparison of Optomap ultrawide-field imaging versus slit-lamp biomicroscopy for assessment of diabetic retinopathy in a real-life clinic
}

\author{
This article was published in the following Dove Press journal: \\ Clinical Ophthalmology \\ 29 July 2014 \\ Number of times this article has been viewed
}

\section{Robert M J Purbrick \\ Shahrnaz Izadi \\ Ankur Gupta \\ $\mathrm{N}$ Victor Chong}

Oxford Eye Hospital, John Radcliffe Hospital, Oxford, UK
Correspondence: N Victor Chong

Oxford Eye Hospital, West Wing, John

Radcliffe Hospital, Oxford, OX3 9DU, UK

Tel +44 I865 234736

Fax +44 I865 23 45I5

Email victor.chong@eye.ox.ac.uk
Purpose: We aimed to assess the agreement between clinical assessment of diabetic retinopathy and Optomap ultrawide-field imaging (UWFI) in a real-life clinic setting.

Methods: Structured examination findings, from diabetic patients attending routine medical retina clinics in July 2011, were retrospectively compared with the grade obtained from Optomap UWFI images, graded independently by two ophthalmologists, taken at the same visit.

Results: A total of 84 eyes (42 patients) were examined, and 74 eyes (37 patients) were suitable for analysis. The hospital Eye Service slit-lamp biomicroscopy grades for retinopathy were: no diabetic retinopathy in zero eyes; background retinopathy in 21 eyes; preproliferative retinopathy in 34 eyes; and proliferative retinopathy in 19 eyes. For retinopathy, the agreement between the Optomap UWFI and clinical grading was moderate for both graders $(\kappa=0.57$ and $\kappa=0.63)$, and there was almost perfect agreement between the two graders $(\kappa=0.92)$. The clinical grades for the presence of photocoagulation scars were: no photocoagulation scars in 46 eyes and photocoagulation scars visible in 28 eyes, indicating substantial agreement between the Optomap UWFI and clinical grading for both graders $(\kappa=0.73$ and $\kappa=0.64)$. There were two instances where proliferative retinopathy was documented clinically but graded as preproliferative by both graders of Optomap UWFI. These were investigated, and neither patient required treatment, ie, the difference in retinopathy grade would not have affected the patient outcomes.

Conclusion: This study demonstrated moderate agreement between Optomap UWFI and hospital slit-lamp biomicroscopy grading of patients' retinopathy in a real-life medical retina clinic setting. The authors believe that Optomap UWFI is, not only a very useful adjunct to clinical examination in terms of detection and recording of retinopathy, but also, could have a role in standalone "virtual" clinics.

Keywords: photography

\section{Introduction}

The Medical Retina service at Oxford Eye Hospital uses the Optos Optomap Panoramic 200Tx ${ }^{\mathrm{TM}}$ (Optos PLC, Dunfermline, UK) digital ultrawide-field imaging (UWFI) system to document objectively the stage of diabetic retinopathy and response to treatment. There are a number of examples in the literature showing that under study conditions, Optomap UWFI is comparable with more conventional retinal imaging techniques ${ }^{1,2}$ as well as mydriatic slit-lamp biomicroscopy, ${ }^{1,3,4}$ in the assessment of diabetic retinopathy.

Optomap UWFI angiography has been shown to improve the detection and classification of diabetic retinopathy. ${ }^{5}$ However, we believe this is the first paper assessing the utility of Optomap UWFI color images in diabetic retinopathy grading alongside a "real-life" clinic situation. 
We report a comparison of National Screening Committee (NSC) grading ${ }^{6}$ using Optomap UWFI with the clinical grading obtained in a routine medical retina clinic staffed by ophthalmologists with various levels of expertise. There is a need for the development of alternative strategies for the assessment of diabetic retinopathy within UK hospital eye services, due to the ever-increasing demands on capacity. ${ }^{7}$

\section{Materials and methods}

In a standard medical retina clinic, doctors with various levels of expertise, using mydriatic slit-lamp binocular indirect ophthalmoscopy, examined 84 eyes of 42 diabetic patients. Clinical findings were recorded using the Diabetic Retinopathy Assessment template within the Medisoft Ophthalmology electronic medical record (EMR) software (Medisoft Limited, Leeds, UK), which requires clinicians to make a structured assessment of the clinical signs of diabetic retinopathy and outputs retinopathy grades according to the Early Treatment Diabetic Retinopathy Study (ETDRS), ${ }^{8}$ the English NSC, ${ }^{6}$ and international $1^{9}$ classifications. The NSC gradings were retrieved retrospectively, and the doctors assessing patients in clinic were unaware of the current study.

Optomap digital UWFI, obtained with the patients' eyes in the primary position, were acquired at the same visit. The instrument is able to obtain wide-field images of approximately 180-200 degrees, and the optical resolution with the instrument used in this study was $1,984 \times 1,984$. In keeping with the scanning laser ophthalmoscope principle, images of high contrast and sharpness are obtained, which show less susceptibility to media opacities than conventional photography. ${ }^{3}$ The two graders viewed the images independently and were blinded to further clinical information. The graders' findings were compared with the clinical retinopathy grade for each eye, as recorded on Medisoft EMR software during two consecutive medical retina clinics that took place in July 2011.

Statistical analysis was performed using PASW Statistics for Windows, Version 18.0 statistical software (IBM Corp, Armonk, NY, USA). Agreement between hospital biomicroscopy grading and the grading of Optomap UWFI was assessed using Cohen's weighted kappa coefficient $(\kappa) .{ }^{10}$ The $\kappa$ coefficient looks at agreement or reliability in categorical variables and is often judged as providing agreement that is: poor when $\kappa<0.00$; slight when $0.00 \leq \kappa \leq 0.20$; fair when $0.21 \leq \kappa \leq 0.40$; moderate when $0.41 \leq \kappa \leq 0.60$; substantial when $0.61 \leq \kappa \leq 0.80$; and almost perfect when $\kappa>0.80$.

Ethical approval was granted to allow the authors to review patients' notes and imaging for the purposes of research. We certify that all applicable institutional and governmental regulations concerning the ethical use of human volunteers were followed during this research.

\section{Results}

This study included 42 patients with diabetes mellitus. The mean age of the patients was 60 years (range 27-84 years). Statistical analysis was possible for 37 of the 42 patients (88\% [74/84] eyes) included in the study. Images were not gradable for three patients (7\%), and two patients (5\%) had incomplete Medisoft diabetic retinopathy grading. A total of 23 of the patients were examined by a consultant (VC), while the remaining 14 patients were examined by nonconsultant doctors, including middle-grade doctors and trainees.

For diabetic retinopathy, the clinical biomicroscopy grades were: no diabetic retinopathy (R0) in zero eyes; background retinopathy (R1) in 21 (28\%) eyes; preproliferative retinopathy (R2) in 34 (46\%) eyes; and proliferative retinopathy (R3) in 19 (26\%) eyes. Grading by grader 1 (RMJP) was in agreement with the clinic grading in 50 eyes (68\%), showed a lower grade in eleven (15\%) eyes, and a higher grade in $13(18 \%)$ eyes (Table 1). Grading by grader 2 (SI) was in agreement with the clinic grading in $54(74 \%)$ eyes, showed a lower grade in eight eyes (11\%), and higher grade in 12 eyes (16\%) (Table 2). Agreement between grader and clinic, using weighted $\kappa$, was moderate for both grader $1(\kappa=0.57,95 \%$ confidence interval [CI] 0.43-0.72) and grader 2 (SI) $(\kappa=0.63,95 \%$ CI $0.49-0.78)$. There was almost perfect agreement between the two graders $(\kappa=0.92$, 95\% CI 0.85-0.99) (Table 3).

With regard to the presence or absence of photocoagulation scars, the clinical biomicroscopy grades were: no photocoagulation scars (P0) in 46 eyes and photocoagulation scars visible (P1) in 28 eyes. Optomap UWFI grading by grader 1 was in agreement with the clinical grading in $65(88 \%)$ eyes,

Table I Cross-tabulation of retinopathy/photocoagulation, comparing clinical grading with Optomap ultrawide-field grading by grader I

\begin{tabular}{|c|c|c|c|c|c|c|c|}
\hline & & \multicolumn{6}{|c|}{ Clinical grading } \\
\hline & & Ro & $\mathbf{R I}$ & $\mathbf{R 2}$ & $\mathbf{R 3}$ & Po & $\mathbf{P I}$ \\
\hline Grader I & Ro & 0 & 1 & 0 & 0 & & \\
\hline \multirow[t]{5}{*}{ (RMJP) } & $\mathbf{R} \mathbf{I}$ & 0 & 7 & 8 & 0 & & \\
\hline & $\mathbf{R 2}$ & 0 & 13 & 26 & 2 & & \\
\hline & R3 & 0 & 0 & 0 & 17 & & \\
\hline & Po & & & & & & 7 \\
\hline & $\mathbf{P I}$ & & & & & & 21 \\
\hline
\end{tabular}

Notes: $\mathrm{R} 0=$ no diabetic retinopathy, $\mathrm{R} \mathrm{I}=$ background retinopathy, $\mathrm{R} 2=$ preproliferative retinopathy, $\mathrm{R} 3=$ proliferative retinopathy, $\mathrm{PO}=$ no photocoagulation scars, $\mathrm{PI}=$ photocoagulation scars visible.

Abbreviations: $\mathrm{P}$, photocoagulation; $\mathrm{R}$, retinopathy. 
Table 2 Cross-tabulation of retinopathy/photocoagulation, comparing clinical grading with Optomap ultrawide-field grading by grader 2

\begin{tabular}{|c|c|c|c|c|c|c|c|}
\hline & & \multicolumn{6}{|c|}{ Clinical grading } \\
\hline & & RO & $\mathbf{R} \mathbf{I}$ & $\mathbf{R 2}$ & R3 & Po & P I \\
\hline Grader 2 & RO & 0 & 0 & 0 & 0 & & \\
\hline \multirow[t]{5}{*}{ (SI) } & $\mathbf{R} \mathbf{I}$ & 0 & 9 & 6 & 0 & & \\
\hline & $\mathbf{R 2}$ & 0 & 12 & 28 & 2 & & \\
\hline & R3 & 0 & 0 & 0 & 17 & & \\
\hline & PO & & & & & & 9 \\
\hline & PI & & & & & & 19 \\
\hline
\end{tabular}

Notes: $\mathrm{R} 0=$ no diabetic retinopathy, $\mathrm{RI}=$ background retinopathy, $\mathrm{R} 2=$ preproliferative retinopathy, $\mathrm{R} 3=$ proliferative retinopathy, $\mathrm{P} 0=$ no photocoagulation scars, $\mathrm{PI}=$ photocoagulation scars visible

Abbreviations: $\mathrm{P}$, photocoagulation; $\mathrm{R}$, retinopathy.

showed a lower grade in seven (9\%) eyes, and a higher grade in two $(3 \%)$ eyes (Table 1$)$. Grading by grader 2 was in agreement with the clinic grading in 62 (84\%) eyes, showed a lower grade in nine (12\%) eyes, and a higher grade in three (4\%) eyes (Table 2). Agreement between grader and clinic was substantial for both grader $1(\kappa=0.73,95 \%$ CI $0.57-0.89)$ and grader $2(\kappa=0.64,95 \%$ CI $0.46-0.82)$ (Table 1$)$. As was the case for retinopathy grading, there was almost perfect agreement between the two graders $(\kappa=0.90,95 \%$ CI $0.80-1)$ with respect to the grading of photocoagulation (Table 3 ).

\section{Discussion}

We compared two people reading Optomap images with a real-life clinic situation involving doctors with varying levels of expertise - the clinical team did not know that this was happening. Other published studies have compared Optomap images with slit-lamp biomicroscopy by a consultant in a clinical trial setting, which is far from the reality of a busy medical retina clinic. ${ }^{3,5}$ Results showed moderate agreement between Optomap UWFI and hospital Eye Service biomicroscopy grading with regard to retinopathy $(\mathrm{R} 0-\mathrm{R} 3)$ and substantial agreement with regard to photocoagulation (P0 or P1).

Table 3 Cross-tabulation of the level of retinopathy/photocoagulation, comparing the graders of the Optomap ultrawide-field images

\begin{tabular}{|c|c|c|c|c|c|c|c|}
\hline & & \multicolumn{6}{|c|}{ Grader 2 (SI) } \\
\hline & & RO & RI & $\mathbf{R 2}$ & R3 & PO & P I \\
\hline Grader I & RO & 0 & I & 0 & 0 & & \\
\hline \multirow{5}{*}{ (RMJP) } & $\mathbf{R} \mathbf{I}$ & 0 & 13 & 2 & 0 & & \\
\hline & $\mathbf{R} 2$ & 0 & I & 40 & 0 & & \\
\hline & $\mathbf{R 3}$ & 0 & 0 & 0 & 17 & & \\
\hline & PO & & & & & & 1 \\
\hline & PI & & & & & & I \\
\hline
\end{tabular}

Notes: $\mathrm{R} 0=$ no diabetic retinopathy, $\mathrm{R} \mathrm{I}=$ background retinopathy, $\mathrm{R} 2=$ preproliferative retinopathy, $\mathrm{R} 3=$ proliferative retinopathy, $\mathrm{P} 0=$ no photocoagulation scars, $\mathrm{PI}=$ photocoagulation scars visible.

Abbreviations: $\mathrm{P}$, photocoagulation; $\mathrm{R}$, retinopathy.
Of the 19 eyes that were graded clinically as R3, 17 (89\%) of these 19 eyes were graded as R3 by the graders and two (11\%) were graded as R2. Clearly, it is important to look at these discrepancies as any "missed" proliferative retinopathy could lead to harm for the patients concerned, so the clinical notes and Medisoft records were reviewed for all R3 patients. Of the two eyes that were graded R3 on Medisoft but as R2 by the Optomap graders, one was documented as "new vessels elsewhere (NVE) with surrounding old-looking retinal hemorrhage - not active"; and the other had "vitreous hemorrhage" written in the notes, but no new vessels were documented on the retinal drawing. Both patients had had previous panretinal photocoagulation, and neither patient required further laser or surgery as a result of the clinic visit in question, ie, the difference in grading would not have altered the patients' management on these occasions; nevertheless, there was the potential for incidents that could have significant impact on patient care. In a study by Sallam et al, ${ }^{11}$ that compared the level of agreement between grading of digital photographs in a diabetic retinopathy screening service and hospital biomicroscopy grading using the same NSC classification, R3 was only recorded by screeners in two of the nine cases that were picked up on clinical examination - it has been hypothesized that the wider field of view in Optomap images (180-200 degrees) could increase the sensitivity to peripheral new vessels compared with two-field screening photographs (two 45-degree fields).,12

The grading of retinopathy from the Optomap images was very consistent between the two graders, with the $\kappa$ for retinopathy grading (R0-R3) and for presence of photocoagulation scars (grading P0 or P1) suggesting almost perfect agreement.

When comparing the clinical grading to that made from Optomap UWFIs by the two graders, there was substantial agreement with regard to photocoagulation (P0 or P1). This is important as the presence or absence of panretinal photocoagulation could affect how a patient with retinopathy graded as R2 or R3 is managed.

However, for retinopathy, the agreement was not so strong. In practical terms, it is important to consider how the retinopathy grading affects the management of patients; for example, patients graded R0 or R1 may be discharged to annual screening in the community, whereas an R2 grading is likely to lead to closer follow up (ie, 6-monthly as opposed to annual review), and a grading of $\mathrm{R} 3$ could result in the patient being offered laser treatment. In addition, the graders of the Optomap images adopted a similar approach to screening, with a goal of producing the most accurate 
retinopathy grade, whereas the approach in clinic tends to be much more practical, and focused on patient outcomes, ie, the need to treat. There were no examples of a two-step discrepancy between the clinical grading and Optomap retinopathy grading (ie, R0 vs R2 or R1 vs R3), which means that a vastly different patient outcome from the two modalities would have been unlikely. The two "missed" R3 patients have already been discussed - neither patient required more laser treatment, so the outcome would have been unaffected by the different grading - but instances where a patient is graded as R2 instead of R1, or vice versa, could affect the patient's management, particularly in terms of the follow-up interval. Examining the discrepancies between the R1 and R2 gradings revealed that grader 1 was in agreement with the clinical grading of R2 for 26 eyes, gave a more severe grading (ie, R2 vs R1) for 13 eyes, and a less severe grading (ie, R1 vs R2) for eight eyes. In the case of grader 2 , there was agreement with regard to $\mathrm{R} 2$ for 28 eyes, the grader recorded a more severe Optomap grading (ie, R2 vs R1) for 12 eyes, and a less severe Optomap grading (ie, R1 vs R2) for six eyes. This suggests a tendency for the imaging grading to be more severe than the clinical grading, which was also demonstrated in a similar study comparing diabetic eye-screening service images at referral with the subsequent clinical grading. ${ }^{11}$ A weakness of the current study is the lack of opportunity for arbitration, ie, the ability to reassess patients where there was disagreement between the clinical and Optomap grading, to establish whether there had been under- or overgrading, and by which modality, in each case. Furthermore, the clinical grading of diabetic retinopathy was performed by ophthalmologists with varying levels of experience in the medical retina clinic, whereas the Optomap image grading was performed by two experienced ophthalmologists, which could have induced misclassification bias.

In conclusion, this study demonstrated moderate agreement between Optomap UWFI and hospital slit-lamp biomicroscopy grading of patients' retinopathy in a real-life medical retina clinic setting, by doctors of varying levels of expertise. The detection of proliferative retinopathy using UWFI compared favorably with other studies, ${ }^{5}$ and there were no obvious instances where discrepancies between UWFI and clinical grading would have significantly or adversely affected patient outcomes. The authors believe that Optomap UWFI is, not only a very useful adjunct to clinical examination in terms of detection and recording of retinopathy, but also, could have a role in standalone "virtual" clinics, where retinopathy is assessed using UWFI and maculopathy by ocular coherence tomography. However, further investigation with a larger study size is required before we can recommend the replacement of clinical examination with Optomap, and ocular coherence tomography-based virtual clinics as the standard of care.

\section{Acknowledgments}

The photographers were Lewis Smith, Jon Brett, and Matthew Robertson.

\section{Disclosure}

The authors report no conflicts of interest in this work.

\section{References}

1. Silva PS, Cavallerano JD, Sun JK, Noble J, Aiello LM, Aiello LP. Nonmydriatic ultrawide field retinal imaging compared with dilated standard 7-field 35-mm photography and retinal specialist examination for evaluation of diabetic retinopathy. Am J Ophthalmol. 2012;154(3):549-559.e2.

2. Kernt M, Hadi I, Pinter F, et al. Assessment of diabetic retinopathy using nonmydriatic ultra-widefield scanning laser ophthalmoscopy (Optomap) compared with ETDRS 7-field stereo photography. Diabetes Care. 2012;35(12):2459-2463.

3. Neubauer AS, Kernt M, Haritoglou C, Priglinger SG, Kampik A, Ulbig MW. Nonmydriatic screening for diabetic retinopathy by ultrawidefield scanning laser ophthalmoscopy (Optomap). Graefes Arch Clin Exp Ophthalmol. 2008;246(2):229-235.

4. Khandhadia S, Madhusudhana KC, Kostakou A, Forrester JV, Newsom RS. Use of Optomap for retinal screening within an eye casualty setting. Br J Ophthalmol. 2009;93(1):52-55.

5. Wessel MM, Aaker GD, Parlitsis G, Cho M, D’Amico DJ, Kiss S. Ultra-wide-field angiography improves the detection and classification of diabetic retinopathy. Retina. 2012;32(4):785-791.

6. Harding S, Greenwood R, Aldington S, et al; Diabetic Retinopathy Grading and Disease Management Working Party. Grading and disease management in national screening for diabetic retinopathy in England and Wales. Diabet Med. 2003;20(12):965-971.

7. Michelotti MM, Abugreen S, Kelly SP, et al. Transformational change: nurses substituting for ophthalmologists for intravitreal injections - a quality-improvement report. Clin Ophthalmol. 2014;8:755-761.

8. Fundus photographic risk factors for progression of diabetic retinopathy. ETDRS report number 12. Early Treatment Diabetic Retinopathy Study Research Group. Ophthalmology. 1991;98(5 Suppl):S823-S833.

9. Wilkinson CP, Ferris FL, Klein RE, et al; Global Diabetic Retinopathy Project Group. Proposed international clinical diabetic retinopathy and diabetic macular edema disease severity scales. Ophthalmology. 2003;110(9):1677-1682.

10. Cohen J. Weighted kappa: nominal scale agreement with provision for scaled disagreement or partial credit. Psychol Bull. 1968;70(4): 213-220.

11. Sallam A, Scanlon PH, Stratton IM, et al. Agreement and reasons for disagreement between photographic and hospital biomicroscopy grading of diabetic retinopathy. Diabet Med. 2011;28(6):741-746.

12. Cheng SC, Yap MK, Goldschmidt E, Swann PG, Ng LH, Lam CS. Use of the Optomap with lid retraction and its sensitivity and specificity. Clin Exp Optom. 2008;91(4):373-378. 
Clinical Ophthalmology

\section{Publish your work in this journal}

Clinical Ophthalmology is an international, peer-reviewed journal covering all subspecialties within ophthalmology. Key topics include: Optometry; Visual science; Pharmacology and drug therapy in eye diseases; Basic Sciences; Primary and Secondary eye care; Patien Safety and Quality of Care Improvements. This journal is indexed on

PubMed Central and CAS, and is the official journal of The Society of Clinical Ophthalmology (SCO). The manuscript management system is completely online and includes a very quick and fair peer-review system, which is all easy to use. Visit http://www.dovepress.com/ testimonials.php to read real quotes from published authors. 